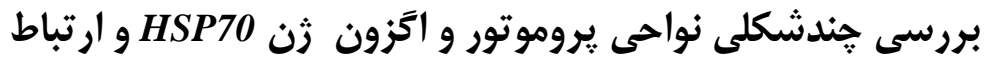

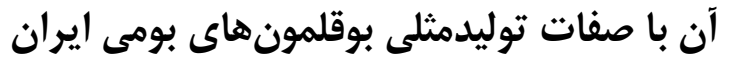

\author{
نيلوفر راستى دوست'، سعيد نيك بين'، بهمن نويد شادَّ قربان الياسى
}

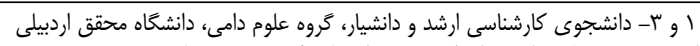

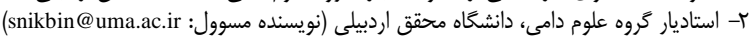

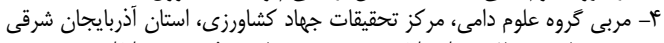

تاريخ دريافت:

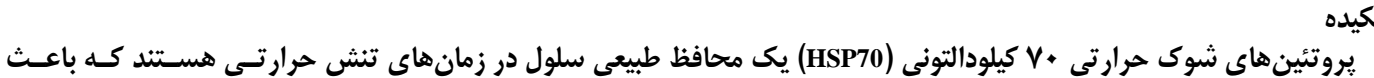

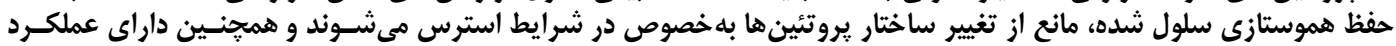

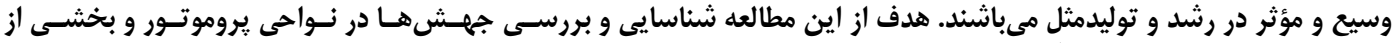

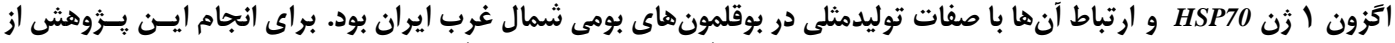

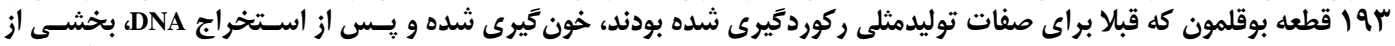

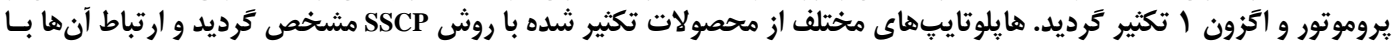

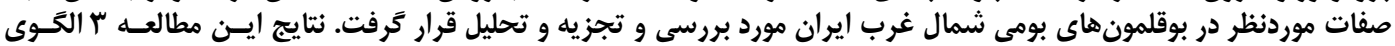

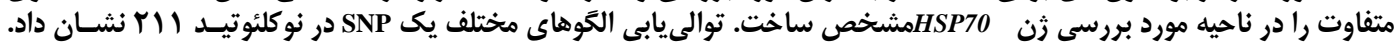

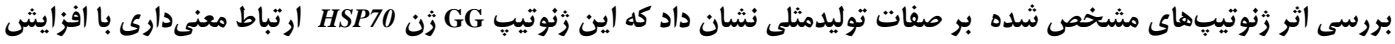

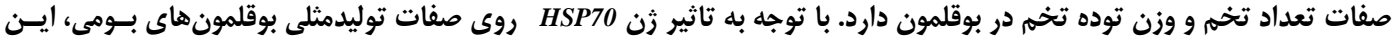

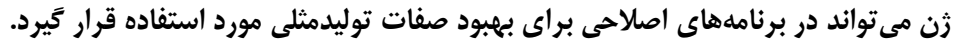

وازههاى كليدى: زن كانديدا، هندشكلى زنى، يروتئين شوك حرارتى، توليد تخم، تفاوتهاى تك نوكلئوتيدى

مىتواند باعث آيِتوز يا مركى سلولى شود. اسييناسى و و

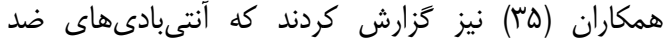

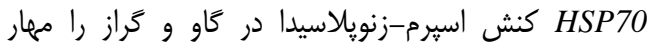
مى كنند. در تحقيقات ديخرى به نقا نقش

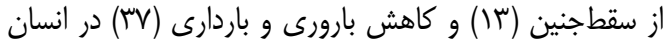

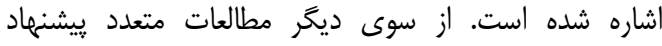

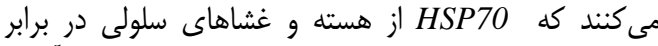

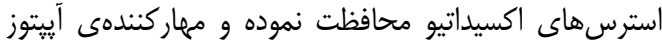

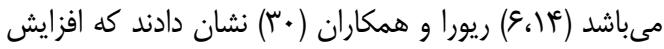

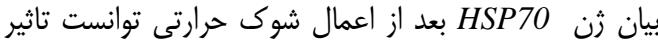

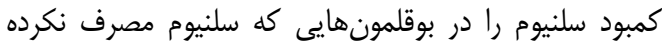

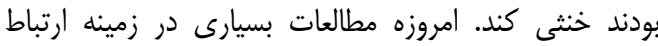

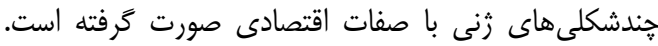

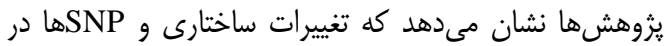

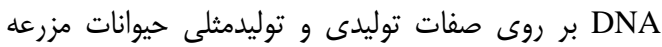

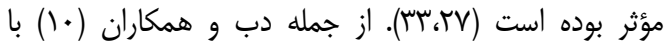

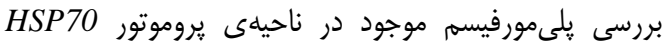

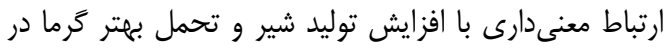

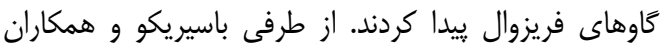

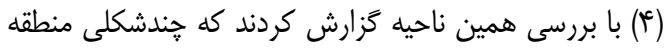

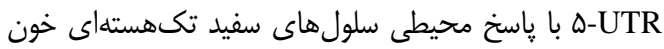

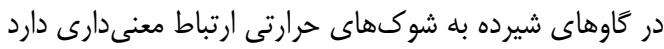

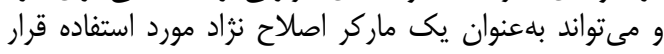

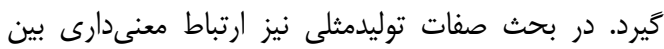

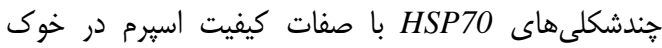

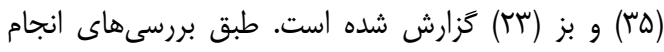

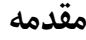

يروتئين شوى حرارتى فراوانترين مولكولهاى داخل

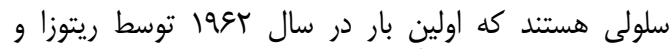

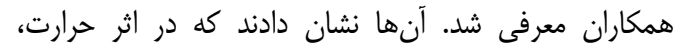

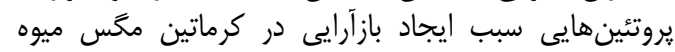

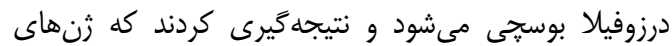

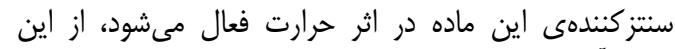

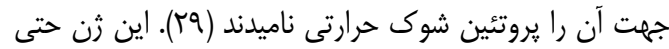

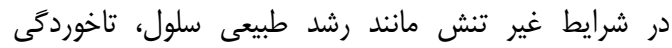

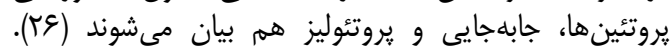

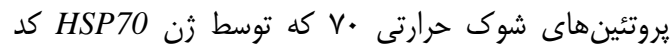
مىشود، از خانوادهى حإيرونها

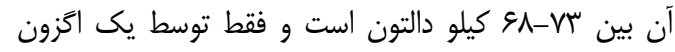

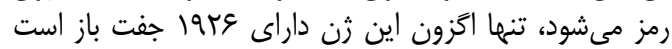

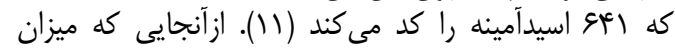

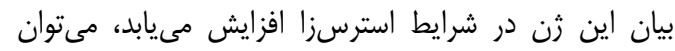

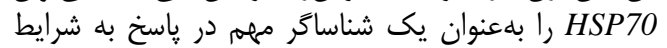

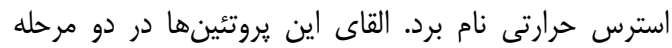

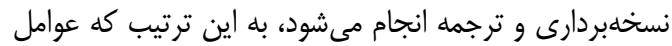

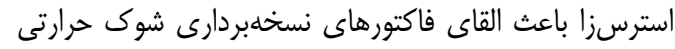

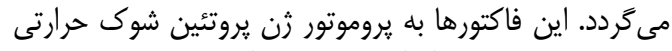

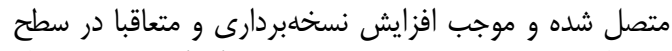

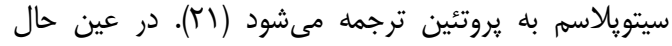

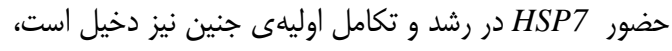

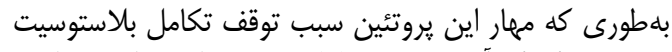
مى كردد (19). آسيبهاى سلولى اينى ناشى از شوك حرارتى تلى 
در بوقلمون از وريد زير بالى صورت گرفته، در لولههاى حاوى إنى

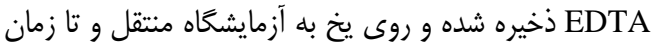

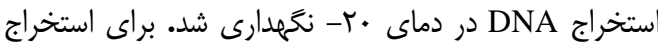
DNA

با توجه به عدم در دسترس بودن ناحيه مورد نظر از ثن

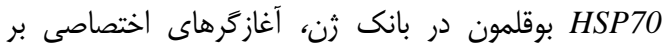

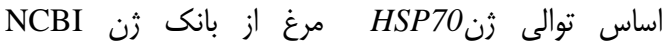

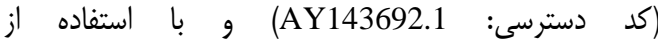

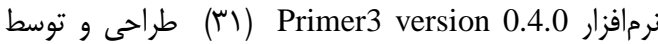

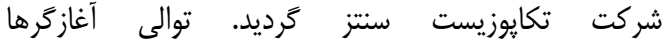

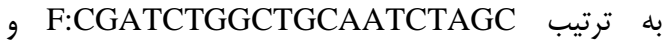
R:GCCCTTTGCCAGACATGATG بررسى يلىمورفيسم ثن HSP70 در بوقلمون قطعهاى از

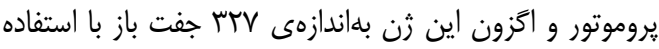

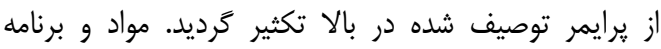
دمايى PCR بلترتيب در جداول ا و ب ارئه شده است.

Table 1. Used materials for PCR of each sample مقدار

$\mid r / D$

t

$1 / 4$

ra

شده، علىرغم وجود مطالعاتى بر روى نواحى مختلف اين ثن

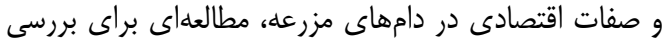

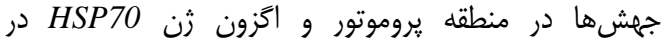

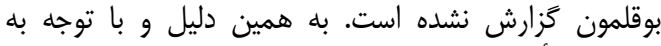

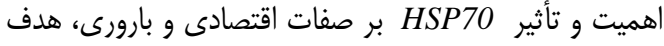

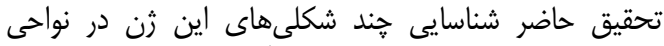

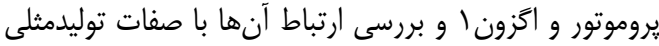

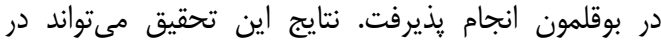
طرحهاى اصلاحنزادى بوقلمون بومى ايران مورد استفاده قرار كيرد.

\section{مواد و روشها}

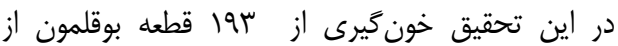

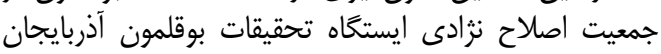

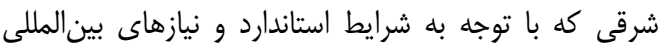

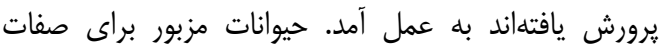

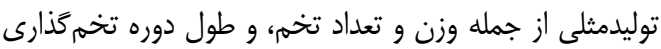

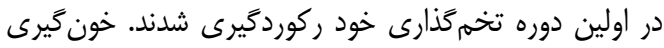

جدول 1- مواد مورد استفاده براى PCR هر نمونه

\begin{tabular}{|c|c|}
\hline 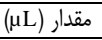 & مواد \\
\hline N/I & آب ديونيزه \\
\hline $\mid r / \Delta$ & مسترميكس (Super PCR MasterMix 2X YT1553) \\
\hline r & DNA الكَو DN \\
\hline $1 / T$ & برايمها \\
\hline TD & حجم نهايى \\
\hline
\end{tabular}

Table 2. PCR program for HSP70 gene

جدول r- برنامه دمايى PCR براى ثن HSP70

\begin{tabular}{|c|c|c|}
\hline زمان & $\left(\mathrm{C}^{\circ}\right)^{\circ}$ & نام مرحله \\
\hline 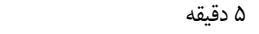 & 94 & كرماى شروع \\
\hline هץ ثانيه & $9 F$ & واسرشت \\
\hline . ب ثانيه & $\Delta r$ & اتصال آغازمر ها \\
\hline . غثانيه. & vr & بسط \\
\hline •ا دقيقه & vr & بسط نهايى \\
\hline
\end{tabular}

تفاوتهاى شناسايى شده در الخوهاى باندى براى در هِيهار

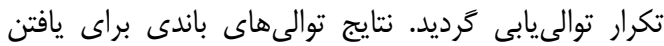

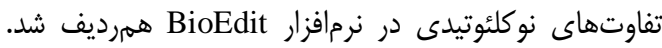

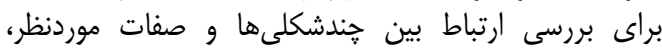

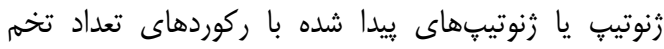

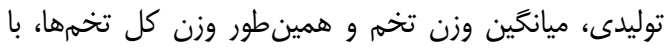

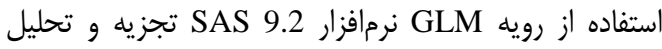
كرديد. مقايسه ميانخينها با روش توفيه توفى در سطح ه درصد

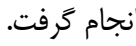

ميانگين، اشتباه معيار كمينه و بيشينه صفات تخمثركارى

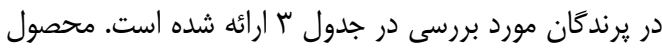

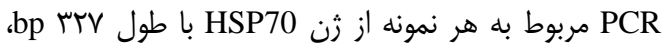

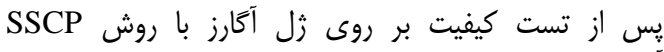

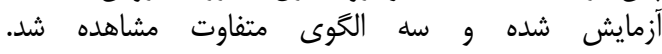

نمونههاى تكثير شده در واكنش زنجيرهى يلىمراز به

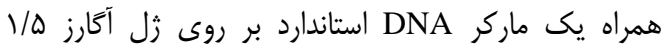

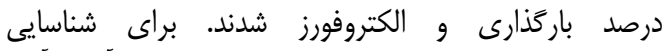

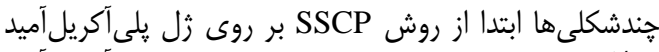

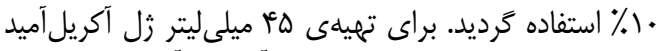

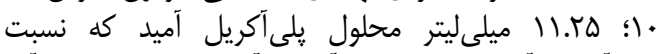

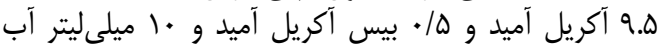

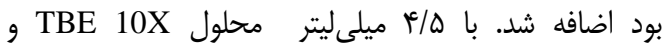

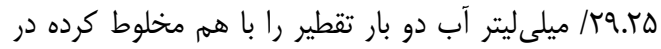

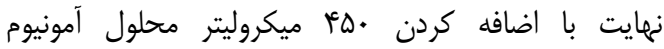

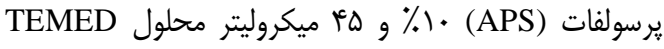

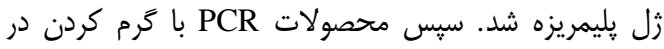

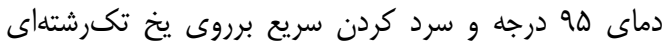

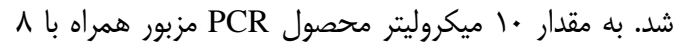

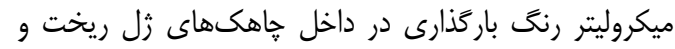

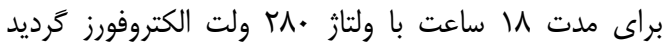


Table 3. Descriptive statistics of egg production treats جلدول r- آمارههاى توصيفى صفات توليد تخمم

\begin{tabular}{|c|c|c|c|c|}
\hline وزن تودمى تخم (5,م) & وزن اولين تخم (5, م) & ميانكين وزن تخم (كرم) & تعداد تخم & \\
\hline rarq/A & $r / q$ & VE/V & $\Delta I / T$ & ميانكين \\
\hline$|f| \%$ & .19 &.$/ 4$ & $1 / 1$ & SE \\
\hline $1 \ldots / 1$ & $9 \mathrm{~V} / \mathrm{V}$ & $98 / 1$ & r & كمينه \\
\hline Veer/v & $\Lambda \cdot / \Delta$ & $N / \Delta$ & qv & بيشينه \\
\hline
\end{tabular}

با توجه به بررسى منابع انجام شده و اين جايياه در بوقلمون

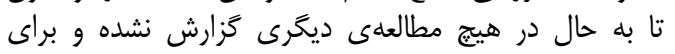

نتايج توالىيابى و همرديف سازى توالىهاى سه الخَّى

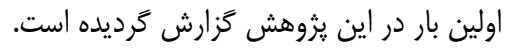

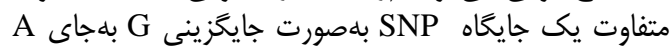

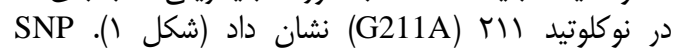
مشاهده شده در ناحيه يروموتور زن HSP70 قرار دار داشت كه

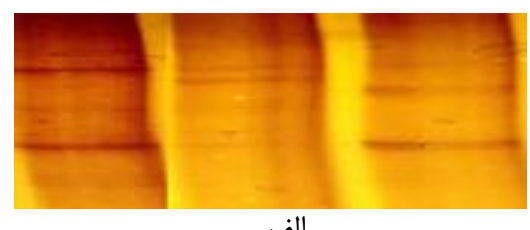

الف
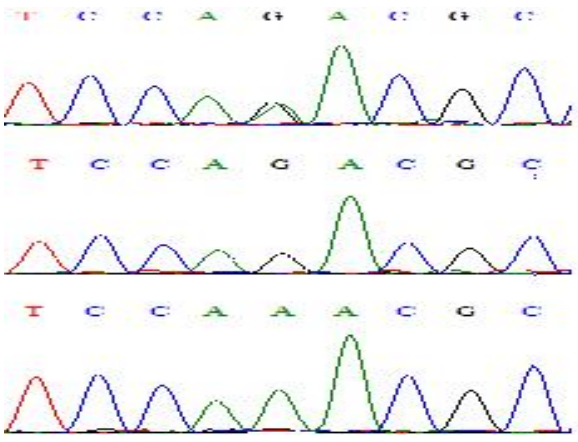

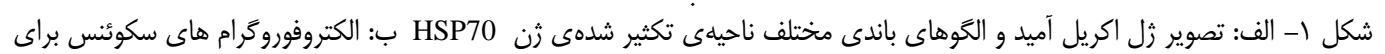

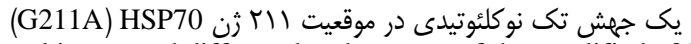

Figure 1. a: Polyacrylamide gel image and different band patterns of the amplified of HSP70 region. b: Sequence electropherograms for one SNP at the amplified HSP70 region, (G211A)

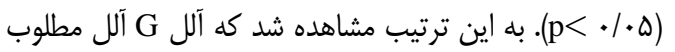

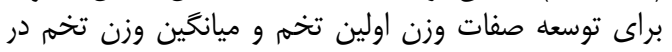
بوقلمونهاى بومى مى باشد.

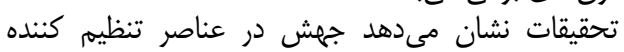

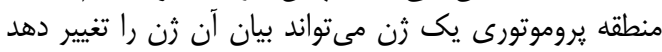

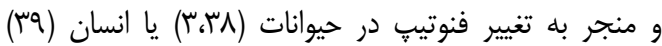

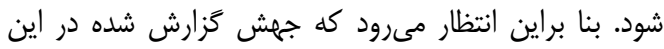

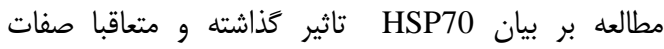

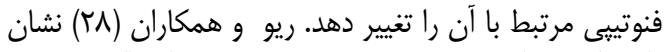

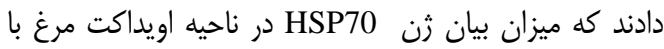

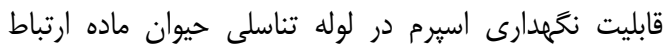

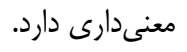

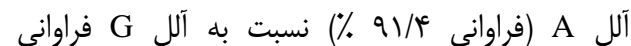

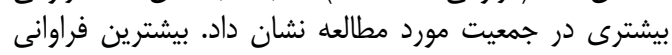

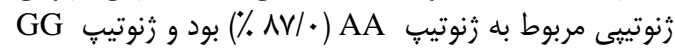

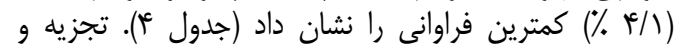

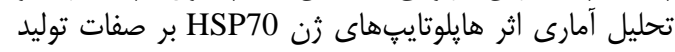

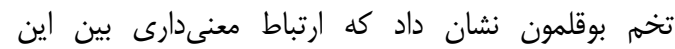

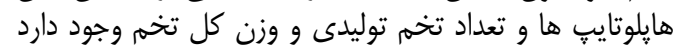

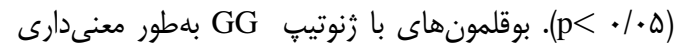

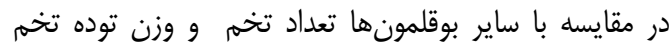

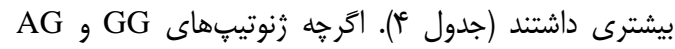

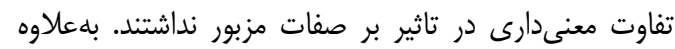

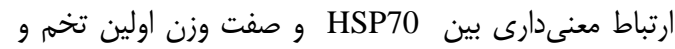
ميانخين وزن تخمم توليدى در مطالعه حاضر مشاهده نشد 


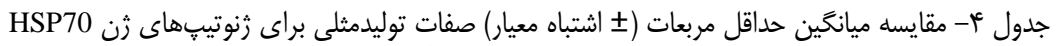
Table 4. Comparison of LS-Mean of ( \pm se) reproductive Traits for HSP70 genotypes

\begin{tabular}{|c|c|c|c|c|c|}
\hline \multicolumn{4}{|c|}{ صفات } & \multirow[b]{2}{*}{ فراوانى } & \multirow[b]{2}{*}{ ز زنوتايب } \\
\hline وزن تودمى تخهم & وزن اولين تخم) & ميانكَين وزن تخم (كَرم) & تعداد تخم & & \\
\hline 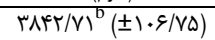 & $\mathrm{VT/ \wedge q}( \pm \cdot / \uparrow \mathrm{\Psi})$ & $V g / V F( \pm \cdot / r q)$ & $\Delta \cdot / 11^{D}( \pm \backslash / \sim \wedge)$ & 191 & $\mathrm{AA}$ \\
\hline$\Delta . \leftarrow \varepsilon / r q^{\mathrm{a}}( \pm \leftarrow \Lambda \cdot / r q)$ & 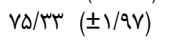 & $\left( \pm I / r T^{\prime}\right) V \varepsilon / r I$ & $\varepsilon q / \cdot .^{\mathrm{a}}( \pm \varepsilon / 4 \cdot)^{\prime}$ & $\wedge$ & GG \\
\hline 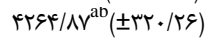 & $\left( \pm 1 / T_{1}\right)$ V & $( \pm \cdot / \wedge q) \vee \varepsilon / 4 \wedge$ & $\Delta \Delta / \mathcal{G})^{\mathrm{ab}}( \pm \psi / / T)$ & iv & $\mathrm{AG}$ \\
\hline . $/$. qq & . / Frq & ./19\% & .1 .48 & & \\
\hline
\end{tabular}

و همكاران (T) ارتباط بين يك جهش حذفى در ناحيهى

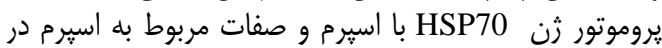

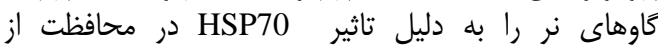

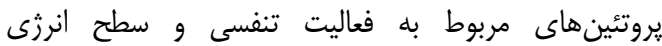

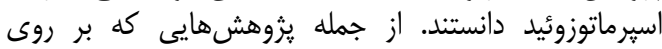

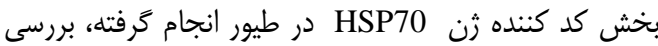

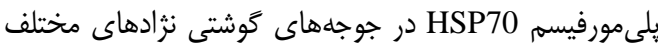

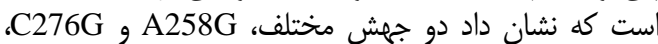

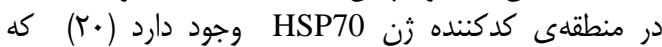

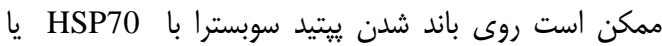

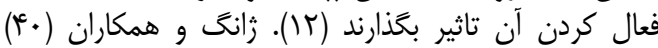

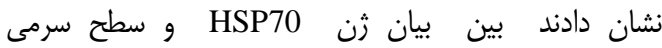
كورتيكوسترون در غازها ارتباط معنى دارى وجود دارن دارد. در يكى سيك

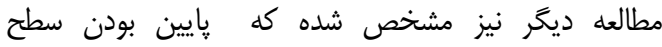

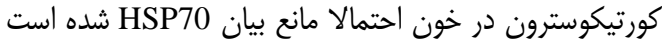

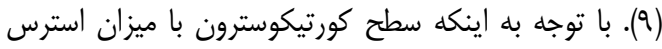

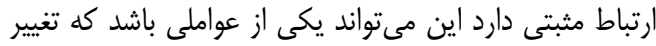

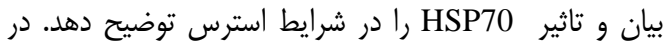

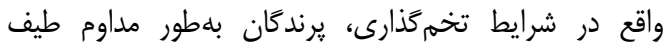

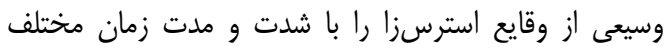

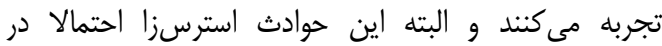

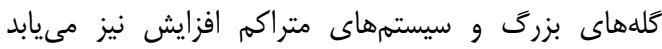

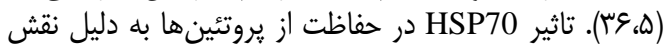

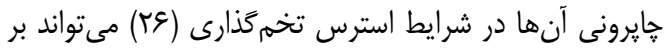

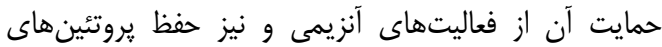

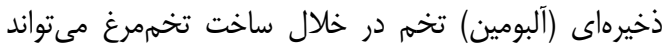

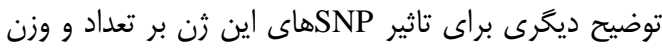
توده تخم توليدى باشد.

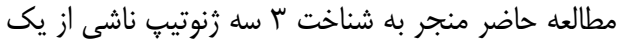

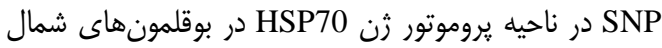

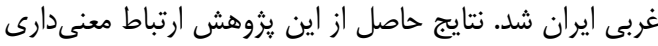

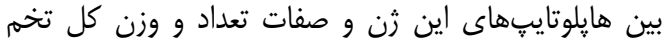

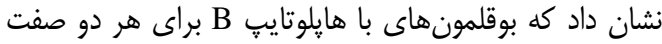

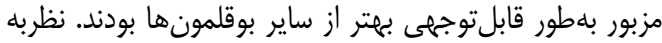

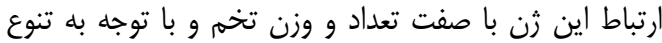

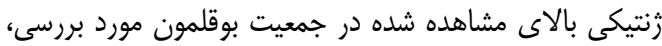

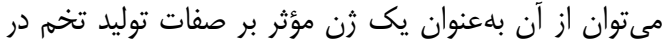

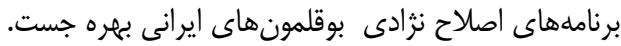

ماريانى و همكاران (IN) كزارش كردند كه ميزان بيان

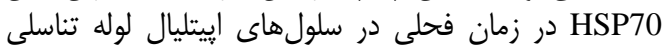

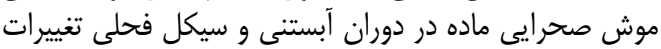

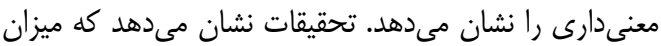

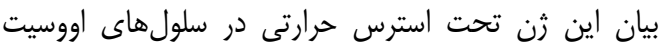

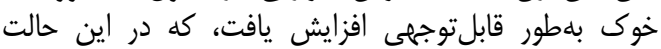

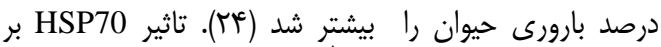

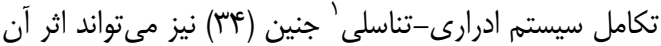

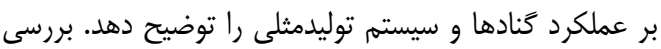

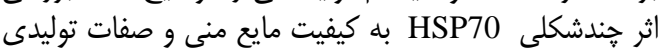

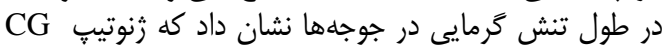

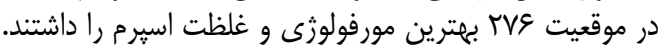

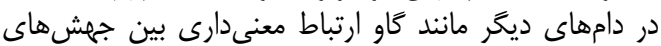

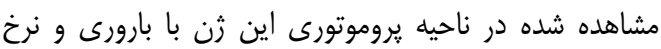

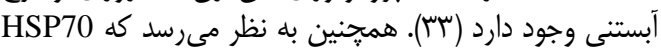

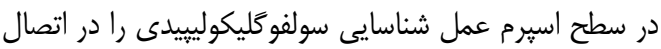

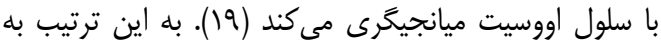

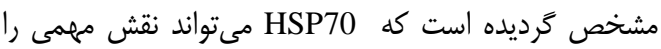

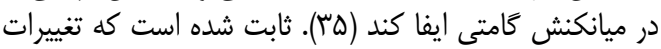

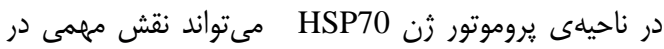

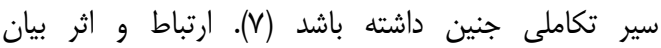

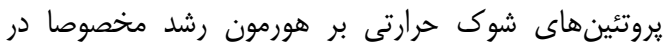

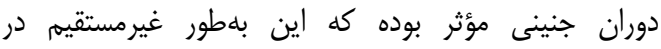

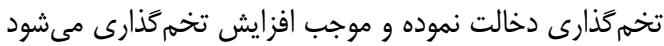

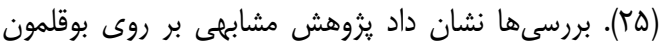

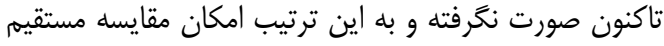

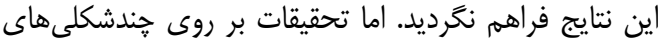

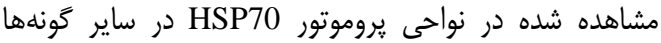

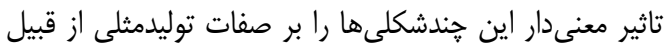

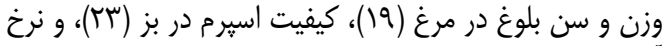

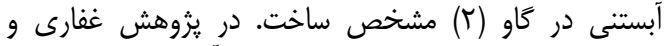

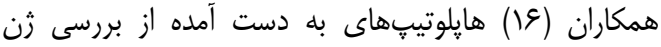

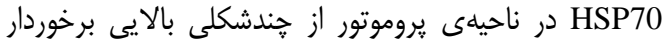

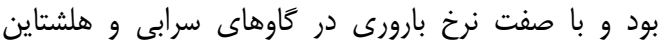

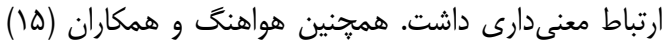

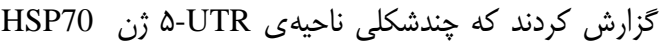

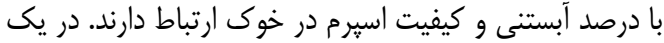

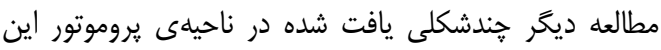

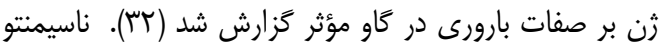


1. Bailes, S.M., J.J. Devers, J.D. Kirby and D.D. Rhoads. 2007. An inexpensive simple protocol for DNA isolation from blood for high-throughput genotyping by polymerase chain reaction or restriction endonuclease digestion. Poultry Science, 86(1): 102-106.

2. Banks, A., M.L. Looper, S. Reiter and C.F. Rosenkrans. 2007. Identification of single nucleotide polymorphisms within the promoter region of the bovine heat shock protein 70 gene and associations with pregnancy. Journal of Animal Science, 85: 12-13.

3. Bai, Y.J.B., Y. Zhang, Y.L. Xue, G. Peng and M.Y. Chen. 2015. Differential expression of CYB5A in Chinese and European pig breeds due to genetic variations in the promoter region. Animal Genetic, 46(1): 16-22.

4. Basiricò, L., P. Morera, V. Primi, N. Lacetera, A. Nardone and U. Bernabucci. 2011. Cellular thermotolerance is associated with heat shock protein 70.1 genetic polymorphisms in Holstein lactating cows Cell Stress and Chaperones, 16(4): 441-448.

5. Blokhuis, H.J., T. Fiks-Van Niekerk, W. Bessei, A. Elson, D. Guemene, J.B. Kjaer, G.A. Levrino, C.J. Nicol, R. Tauson, C.A. Weeks and H.A. Van De Weerd. 2007. The LayWel project: welfare implications of changes in production systems for laying hens. World's Poultry Science Journal, 63: 101-114.

6. Burkat, V., H. Liu, K. Bellmann, D. Wissing, M. Jäättela and M.G. Cavallo. 2000. Natural resistance of human beta cells toward nitric oxide is mediated by heat shock protein 70 . Journal of Biological Chemistry, 275(26): 19521-19528.

7. Chen, B., T. Jia, R. Ma and B. Zhang. 2011. Evolution of hsp70 gene expression: a role 10.for changes in AT-richness within promoters. Public Library of Science, 6: e20308.

8. Dang, X.Y., W.W. Chu, H.C. Shi, S.G. Yu, H.Y. Han and S.H. Gu. 2015. Genetic variants in ABCA1 promoter affect transcription activity and plasma HDL level in pigs. Gene, 555(2):414-420.

9. Davis, K.A., J.B. Schmidt, R.M. Doescher and D.G. Satterlee. 2008. Fear responses of offspring from divergent quail stress response line hens treated with corticosterone during egg formation. Poultry Science, 87: 1303-1313.

10. Deb, R., B.U. Sajjanar, S. Mesh-Singh, R. Singh and G. Sengar. 2013. Promoter variants at AP2 box region of Hsp70.1 affect thermal stress response and milk production traits in Frieswal cross bred cattle. GENE, 532(2): 230-235.

11. Gade, N., P.K. Mahapatra, A. Sonawane, V.K .Singh, R. Doreswamy and M. Saini. 2010. Molecular characterization of heat shock protein 70-1 geneof goat (Capra hircus). Journal of Molecular Biology, $108429,7 \mathrm{pp}$

12. Gan, J.K., L.Y. Jiang, L.N. Kong, X.Q. Zhang and Q.B. Luo. 2015. Analysis of genetic diversity of the heat shock protein 70 gene on the basis of abundant sequence polymorphisms in chicken breeds. Genetics and Molecular Research, 14(1): 1538-1545.

13. Güvenc, M., H. Güven, F. Karate, D.A. Aygun and S. Bekta. 2002. Low levels of selenium in miscarriage. Journal of Trace Elements in Experimental Medicine, 15: 97-101.

14. Hooper, P.L. and J.J. Hooper. 2005. Loss of defense against stress: Diabetes and heat shock proteins. Diabetes Technology and Therapeutic, 7: 204-208.

15. Huang, S.Y., M.Y. Chen, E.C. Lin, H.L. Tsou, Y.H. Kuo, C.C. Ju and W.C. Lee. 2002. Effects of single nucleotide polymorphisms in the 5-flanking region of heat shock protein 70.2 gene on semen quality in boars. Animal Reproduction Science, 70: 99-109.

16. Ghaffari, M., M. Sadeghi, A. Nejati-Javaremi, M. Moradi-Shahrebabak and R. Faraji. 2012. Study of polymorphism in the bovine HSP70 gene promote and its association with conception rate in Holstein and Sarabi breeds. Journal of Agricultural Biotechnology, 5(4): 83-95 (In Persian).

17. Liang, H.M., D.Y. Lin, Y.D. Hsuuw, T. Huang, H.L. Chang, C.Y. Lin, H.H. Wu and K.H. Hung. 2016. Association of heat shock protein 70 gene polymorphisms with acute thermal tolerance, growth, and egg production traits of native chickens in Taiwan. Archives Animal Breeding, 59(2): 173-181.

18. Mariani, M.L., M. Souto, M.A. Fanelli and D.R. Ciocca. 2000. Constitutive expression of heat shock proteins hsp25 and hsp70 in the rat oviduct during neonatal development, the oestrous cycle and early pregnancy. Journal of Reproduction and Fertility, 120: 217-223.

19. Matwee, C., M. Kamaruddin, D.H. Betts, P.K. Basrur and W.A. King. 2001. The effects of antibodies to heat shock protein 70 in fertilization and embryo development. Molecular Human Reproduction, 7 : 829-37.

20. Mazzi, C., O. Aparecido, J. Ferro, F. Tiraboschi, M. Ferro, V. Savino, A. Coelho and M. Macari. 2003. Polymorphism analysis of the hsp70 stress gene in Broiler chickens (Gallus gallus) of different breeds. Genetics and Molecular Biology, 26(3): 275-281.

21. Morimoto, R., I. Kline, M.P. Bimston and D.N. Cotto. 1997. The heat-shock response regulation and function of heat-shock proteins and molecular chaperones. Essays in biochemistry, 32: 17-29.

22. Nascimento, J.M., L.Z. Shi, J. Tam, C. Chandsawangbhuwana, B. Durrant, E.L. Botvinick and M.W. Berns. 2008. Comparison of glycolysis and oxidative phosphorylation as energy sources for mammalian sperm motility, using the combination of fluorescence imaging, laser tweezers and realtime automated tracking and trapping. Journal of Cellular Physiology, 217: 745-751.

23. Nikbin, S., J.M. Panandam, H. Yaakub, M. Murugaiyah and A.Q. Sazili. 2014. Novel SNPs in heat shock protein 70 gene and their association with sperm quality traits of Boer goats and Boer crosses. Animal Reproduction Science, 146(3-4): 176-181. 
24. Pennarossa, G., S. Maffei, M.M. Rahman, G. Berruti, T.A. Brevini and F. Gandolfi. 2012. Characterization of the constitutive pig ovary heat shock chaperone machinery and its response to acute thermal stress or to seasonal variations. Biology of Reproduction, 16, 87(5):1-19.

25. Pitts, G.R., O. Youngren, M. Phillips and R.E. El Halawani. 1996. Photoperiod mediates the ability of serotonin to release prolactin in the turkey. General and comparative endocrinology, 104(3): 265-272.

26. Radons J. 2016. The human HSP70 family of chaperones: where do we stand? Cell Stress and Chaperones, 21: 379-404.

27. Raufian, P., J. Shodja Ghyas, R. Jafari, G. Moghaddam and A. Javanmard. 2018. Identification of Genetic Variation in two Candidate Genes of TLR2 and TNF $\alpha$ and its Association with Mastitis in Holstain Cattle. rap Journal of Research on Animal Production, 8(18):147-154.

28. Riou, C., M. Saint-Dizier and N. Gerard. 2015. 146 Sperm Storage: Expression OF Progesterone Receptor, Structural Protein, AND Heat Shock Protein In the avian oviduct. Reproduction Fertility and Development, 28(2): 203-230.

29. Ritossa, F.A. 1962. New puffing pattern induced by temperature shock and DNP in drosophila. Cellular and Molecular Life Sciences, 18(12): 571-573.

30. Rivera, R.E., V.L. Christensen, F.W. Edens and M.J. Wineland. 2005. Influence of selenium on heat shock protein 70 expression in heat stressed turkey embryos (Meleagrisgallopavo). Comparative biochemistry and physiology Part A, Molecular and integrative physiology, 142(4): 427-432.

31. Rozen, S. and H.J. Skaletsky. 2000. Krawetz S, Misener S (eds) Bioinformatics Methods and Protocols: Methods in Molecular Biology. Humana Press, Totowa, NJ, pp: 365-386.

32. Rosenkrans, C.H., J.R.A. Banks, S. Reiter and M. Looper. 2010. Calving traits of crossbred Brahman cows are associated with Heat Shock Protein 70 genetic polymorphisms. Animal Reproduction Science, 119: 178-182.

33. Sadeghi, M., M. Moradi Shahr Babak, G. Rahimi Mianji and A. Nejati Javaremi. 2010. Effect of STAT5A Gene Polymorphism on Milk Production Traits in Iranian Holstein Bulls Journal of Research on Animal Production, 1(2): 37-44.

34. Shi, W., G. Xu, C. Wang, S.M. Sperber, Y. Chen, Q. Zhou, Y. Deng and H. Zhao. 2015. Heat Shock $70-\mathrm{kDa}$ Protein 5 (Hspa5) Is Essential for Pronephros Formation by Mediating Retinoic Acid Signaling. Journal of Biological Chemistry, 290: 577-589.

35. Spinaci, M., S. Volpe, C. Bernardini, M. De. Ambrogi, C. Tamanini and E. Seren. 2005. Immunolocalization of heat shock protein 70 (Hsp70) in boar spermatozoa and its role during fertilization. Molecular Reproduction and Development, 72: 534-41.

36. Solomon, S.E. 2002. The oviduct in chaos World's Poultry Science Journal, 58: 41-48.

37. Tan, H., Y. Xu, J. Xu, F. Wang, S. Nie, M. Yang, J. Yuan, R.M. Tanguay and T. Wu. 2007. Association of increased heat shock protein 70 levels in the lymphocyte with high risk of adverse pregnancy outcomes in early pregnancy: a nested case-control study. Cell Stress Chaperones, 12: 230236.

38. Yu, S., J. Liao, M. Tang, Y. Wang, X. Wei, L. Mao and C. Zeng. 2017. A functional single nucleotide polymorphism in the tyrosinase gene promoter affects skin color and transcription activity in the black-boned chicken. Poultry Science Journal, 96(11): 4061-4067.

39. Yin, J., J. Xie, S. Liu, H. Zhang, L. Han, W. Lu, Q. Shen, G. Xu, H. Dong, J. Shen, J. Zhang, J. Han, L. Wang, Y. Liu, F. Wang, J. Zhao, Q. Zhang, W. Ni, H. Wang and G. Cao. 2011. Association between the various mutations in viral core promoter region to different stages of hepatitis B, ranging of asymptomatic carrier state to hepatocellular carcinoma. The American journal of gastroenterology, 106(1): 81-92.

40. Zhang, W., X. Xiao, J.K. Gan, X.Q. Zhang, L.N. Kong and Q.B. Luo. 2015. Characterization of HSP70 and its expression in tissue: correlation with physiological and immune indices in goose (Anser cygnoides) serum Genetics and Molecular Research, 14(4): 12288-12298. 


\title{
Investigation of Polymorphisms in Promoter and Exon Regions of HSP70 Gene and Their Associations with Reproduction Traits of Indigenous Turkeys of Iran
}

\author{
Niloofar Rastidoust ${ }^{1}$, Saeid Nikbin ${ }^{2}$, Bahman Navidshad $^{3}$ and Ghorban Elyasi ${ }^{4}$ \\ 1 and 3- M.Sc. Student and Associate Professor, Department of Animal Science, University of Mohaghegh Ardabili \\ 2- Assistant Professor, Department of Animal Science, Mohaghegh Ardabili University \\ (Corresponding author: snikbin@uma.ac.ir) \\ 4- Trainer of Animal Science Department Agricultural Jahad Research Center, East Azarbaijan \\ Received: January 27, 2018 \\ Accepted: September 22, 2018
}

\begin{abstract}
Heat shock proteins of $70 \mathrm{kDa}$ (HSP70) are a natural protector of the cell during heat stress through maintaining cell homeostasis and preventing proteins from denaturation, especially in stressed conditions. In addition, HSP70 widely influence growth and reproduction traits. The present study objected to identify polymorphisms in regions of promoter and part of exon 1 of HSP70 gene and their association with reproductive traits in indigenous turkeys of northwestern Iran. The blood samples of 193 turkeys recorded for reproduction traits, were taken and after DNA extraction, promoter and exon 1 regions were amplified. Various haplotypes were identified by SSCP method and their association with the traits in the turkeys analyzed. The results of this study identified 3 different pattern in the investigated regions of the HSP70 gene. Sequencing the different haplotype pattern revealed a single nucleotide polymorphism (SNP) in $211 \mathrm{bp}$. The Statistical analysis of the effect of the genotypes of $H S P 70$ on the reproduction traits showed that the GG genotype of this gene had a significant association with higher egg number and total egg mass of turkeys. Regarding the effect of the HSP70 gene as an effective gene on the reproductive traits of the indigenous turkeys, it can be used in breeding programs to improve reproductive traits and egg production in the indigenous turkey population.
\end{abstract}

Keywords: Candidate gene, Polymorphism, Heat shock proteins, Egg production, Single nucleotide polymor 